\title{
Attacking the system
}

\author{
Next-generation auto-immune therapies target pathways rather than symptoms
}

\author{
Philip Hunter
}

A

uto-immune diseases are a major health problem in both developed and developing countries: They cause as many deaths as the leading infectious diseases and exact an even greater toll on patients' quality of life given their chronic nature (Box 1). Reliable data for all auto-immune diseases are hard to collect, but a 2011 study by the American Autoimmune Related Diseases Association (AARDA) reported that the total annual cost of just seven leading auto-immune diseases (Crohn's disease, ulcerative colitis, systemic lupus erythematosus (SLE), multiple sclerosis (MS), rheumatoid arthritis (RA), psoriasis, and scleroderma) was somewhere between US $\$ 51.8$ billion and US $\$ 70.6$ billion in the USA alone (http://www.diabetesed.net/page/_ files/autoimmune-diseases.pdf). The AARDA report also drew attention to the indirect societal toll with one example being sufferers from RA in the USA, who experienced a decline in average earnings from US $\$ 18,409$ to US $\$ 13,900$ per year, and the number of jobs they were able to perform dropped from 11.5 to 2.6 million. It was also found that approximately 50 percent of RA patients were unable to work at all within ten years after disease onset.

\section{"What makes the impact of auto-immune diseases particularly damaging is that curative treatments have mostly proved elusive."}

The prevalence and impact of auto-immune diseases have risen during the past decades: The AARDA estimates that 50 million people in the USA alone suffer from an auto-immune disease (http://www.aarda.org/autoimmuneinformation/ autoimmune-statistics/), while an earlier report from the US National Institutes of Health estimated that 23.5 million people are affected. The difference in the numbers is owing to the increasing number of autoimmune diseases recognized by medical science: more than 80 diseases now, compared to the 24 or so that were used as the basis for the NIH estimate, according to the AARDA website (same reference). There is also strong evidence that the incidence of at least some major auto-immune diseases is increasing, especially in developed nations: For example, a number of studies have found that type 1 diabetes has been on the rise for a century. What makes the impact of autoimmune diseases particularly damaging is that curative treatments have mostly proved elusive. Auto-immune diseases can rarely if ever be cured; instead, their progression can be controlled and the symptoms alleviated, although there is some potential to achieve remission for long periods with low levels of symptoms.

$\mathrm{T}$ he major challenge for efficient therapy is that auto-immune diseases are complex and usually involve numerous genetic and environmental factors There is no pathogen to attack and no clearcut immunological or genetic factors, proteins, or metabolic or regulatory pathways that can be targeted with drugs. To date, treatments have therefore relied on broad-spectrum drugs that target inflammatory systems, but with mixed results that vary significantly between diseases.

One of the few real success stories is the treatment of RA, the root cause of which is not yet fully understood. It has been known for some time, however, that one characteristic of RA is upregulation of TNF (tumor necrosis factor)-dependent signaling, which regulates the activity of various immune cells. This knowledge led to the identification of anti-TNF drugs that alleviate the symptoms of RA [1]. One of the most successful drugs, infliximab, was actually first approved for Crohn's disease, another auto-immune condition, in 1998. Infliximab is a monoclonal antibody against TNF-alpha, which prevents the cytokine from binding to its target receptor. Anti-TNF drugs in general have proved unexpectedly successful against RA, according to Tim Vyse, Professor of Molecular Medicine specializing in Lupus disease at King's College in London, UK: "Rheumatoid arthritis was one of the early beneficiaries of anti-inflammatory drugs in the sense that anti-TNF had an incredible effect and got the game going," he said. But he pointed out that this success has turned out to be a mixed blessing, as it created unrealistic expectations that drugs could have a similar impact on other auto-immune diseases.

\section{"To date, treatments have therefore relied on broad-spectrum drugs that target inflammatory systems, but with mixed results that vary significantly between diseases"}

One of the problems is that it is still not clear precisely why anti-TNF works so well against RA. "There's an irony there in that the biologic evidence for its efficacy was not 


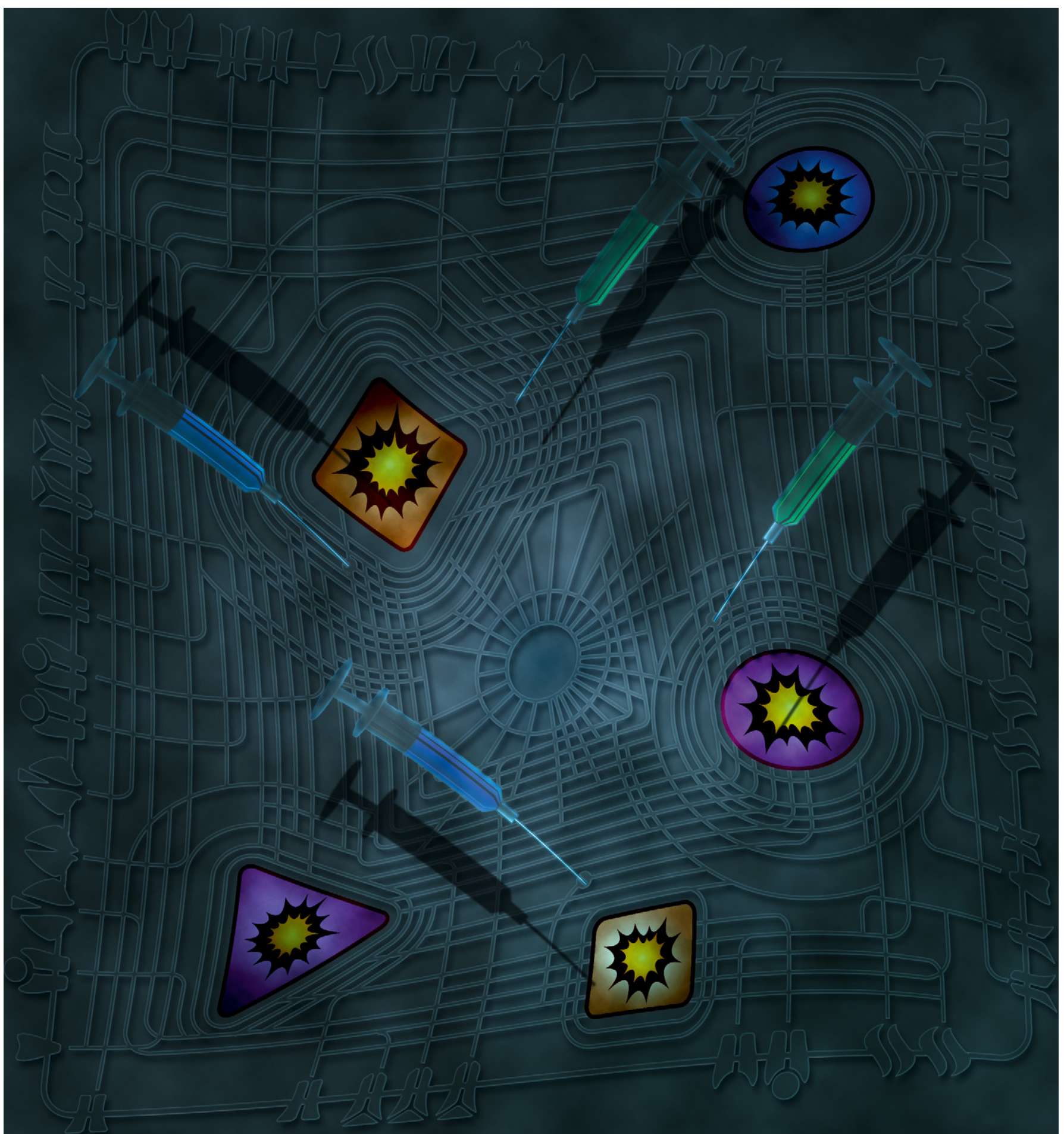

strong," Vyse explained. "It was as much luck as judgment that TNF worked. People still can't answer the question of why antiTNF is so effective." Part of the puzzle is that the immune system has redundant pathways that can take over if one part is knocked out, but this does not seem to happen in many cases of RA. "It's a bit surprising that targeting one molecule has such a big effect," Vyse said.

A lack of knowledge about how a drug works increases the risk of serious unforeseen side effects, however. According to Chris Cotsapas, Assistant Professor of Neurology and Genetics at the Yale School of Medicine, who focuses on MS: "If you treat rheumatoid arthritis patients with no history of MS [...] with anti-TNF, the rheumatoid arthritis recedes, but they then tend to develop a neurological deficit [...] If you stick them in an MRI (Magnetic Resonance Imaging) machine, you can see clearly that they have developed MS. You don't see it until the treatment has been administered, so it is not a 


\section{Box 1: Some common auto-immune diseases}

\section{Graves' Disease}

The most common auto-immune disease in which the body produces antibodies to the receptor for thyroid-stimulating hormone (TSH). It usually attacks the thyroid, frequently causing it to enlarge to twice its size or more (goiter) and become overactive, with related hyperthyroid symptoms such as increased heartbeat, muscle weakness, disturbed sleep, and irritability. No ideal cure but it can be controlled, with last resort surgical excision of the gland. Other treatments include anti-thyroid drugs, which reduce the production of thyroid hormone, and radioiodine to shrink the gland and reduce its activity.

\section{Hashimoto's thyroiditis}

Another auto-immune disease of the thyroid gland that involves a variety of cell- and antibody-mediated immune processes. It was the first disease to be recognized as auto-immune in 1912. In this case, the gland's activity is reduced; treatment involves thyroid hormone replacement agents such as levothyroxine, triiodothyronine, or desiccated thyroid extract. In most cases, the treatment has to be taken for the rest of the patient's life, but effectively controls the disease.

\section{Vitiligo}

This disease causes pale patches to develop on the skin owing to lack of melanin resulting from auto-immune attack on melanocytes. No perfect cure is available, but steroid creams reduce the effect, while phototherapy can work even better, albeit with some risk of causing skin cancer. A synthetic hormone analog called afamelanotide restoring some melanin production is in phase II and III clinical trials for vitiligo.

\section{Pernicious anemia}

Involves auto-immune destruction of gastric parietal cells, causing loss of B12 production. Can be treated quite well with various forms of B12 supplementation.

\section{Glomerulonethritis}

This disease is characterized by damage to kidney filters - the glomeruli-often through inflammation of the glomerular small blood vessels resulting from auto-immune activity. Can be alleviated by changes to diet, such as eating less salt and drugs to lower blood pressure, such as angiotensinconverting enzyme (ACE) inhibitors, to reduce the strain on the kidneys. Immunosuppressants are also been used.

\section{Crohn's Disease}

This type of inflammatory bowel disease (IBD) may affect any part of the gastrointestinal tract from mouth to anus. Not always defined as an autoimmune disease, but there is growing evidence that it shares some of the same pathways in many cases and therefore will respond to the same classes of immune system modulators. Some anti-TNF drugs used for RA have also shown some promise against Crohn's.

Up till now, there is no satisfactory medication in most cases, with treatment comprising lifestyle and dietary changes, along with measures to reduce stress and exercise. Some cases may also be treatable by long-term antibiotic courses to control bacterial activity that otherwise sustains the inflammatory response [4]

\section{Type 1 Diabetes}

The disease is usually diagnosed after auto-immunity has already destroyed the insulin-producing beta cells in the pancreas. Treatment is therefore dealing with the consequence through lifetime insulin injections, which usually manages the condition quite well, but requires a controlled diet and exercise to modulate blood glucose levels. There is growing hope that stem cell-derived beta cells can effect a permanent cure, avoiding the need for insulin injections, which do not yield totally normal glucose metabolism.

\section{Multiple Sclerosis (MS)}

This is an auto-immune attack on the central nervous system (CNS) and specifically the myelin sheath insulating brain, spinal cord, and optic nerves. The disease is rarely fatal itself, so research has focused on drugs that reduce the incidence and severity of the attacks that punctuate periods of remission.

\section{Rheumatoid Arthritis (RA)}

Second most common auto-immune disease that causes inflammation and damage to the joints. It has shown good response to anti-TNF drugs (see main article).

\section{Systemic Lupus Erythematosus}

An auto-immune disease in which the body's immune system attacks tissue in multiple organs. To date, only broad-spectrum steroids have proved helpful, but there is hope and growing evidence that drugs targeting the interferon system could be effective in greatly reducing symptoms (see main article).

case of people being predisposed to both. So clearly there is a lot of subtlety and complexity there that we don't understand yet."

Although only a minority of RA patients on anti-TNF develop MS, it highlights the shortcomings of the prevailing treatments for many auto-immune diseases, despite individual successes against the target condition. "For autoimmune diseases, most therapies chase the proximal consequence of pathology, the immune response, and knock it down, but they don't address the underlying deficit," Cotsapas explained. "The reason you care about that is that if you hit a walnut with a hammer, you rarely have a whole nut left." Yet Cotsapas is optimistic that the current generation of broad-spectrum immune suppressants, such as steroids, will eventually be substituted with more specific treatments that can be tailored to a particular condition. Indeed, recent research has 
shown that most auto-immune diseases share many genetic loci [2].

$\mathrm{T}$ here is still a lot of work to do mapping these loci to specific transcription pathways and to different conditions. A key point is that each pair of auto-immune diseases share a different set of common loci, but each disease also has other loci that are shared with other conditions. "[B]ecause the genetics are shared it looks like some of the pathways are shared," Cotsapas said. "So given the molecular insight we can gain from the genetics, one question for us is: can we use it to develop new drugs and decide which patients should receive those drugs?"

\section{"Improved knowledge of the pathways involved should help physicians to reduce the risk of side-effects and better tailor drug regimes to individual patients"}

The insight that auto-immune diseases have genetic loci in common explains why a given drug can sometimes be used to treat more than one disease. Infliximab is now prescribed for sufferers of several autoimmune diseases including ulcerative colitis as well as RA and Crohn's disease (http://www.drugs.com/news/fda-expandsremicade-indication-ulcerative-colitis-1943. html). It may also explain some of the side effects, including the effect of causing one auto-immune disease while treating another: "If you block some part of the immune system, for example with TNF receptor antagonism, there might be some other compensatory mechanisms, especially in some people, that might release susceptibility to some auto immune diseases," Vyse explained. "My understanding is that that's quite uncommon though."

Improved knowledge of the pathways involved should help physicians to reduce the risk of side effects and better tailor drug regimes to individual patients. The goal is not to personalize treatments to the patient's specific genetics, but more to the specific pathology of the disease. "The game is twofold in terms of how far genetics can be useful for therapy development," Cotsapas said. "One, can we figure out which patient should receive which therapy. [..] So instead of having this one-size-fits-all, if this patient looks like this, we should try therapy $\mathrm{X}$ first. The genetics here is telling us there are multiple pathways associated with increased risk, and if we can understand what those pathways are, we can understand what the risk factors for that disease are and see if the patient has that deficit."

$\mathrm{t}$ might also make research into drugs for auto-immune diseases more viable for pharmaceutical companies if they can be used for multiple diseases. "The economics of auto-immune drug development can become quite challenging," Cotsapas commented. "If a drug targets 1 in 1,000 people and you then slice that population into quarters or fifths, that becomes a small market share. If, however, that therapy is useful for $25 \%$ rheumatoid arthritis patients and $10 \%$ of MS patients and $35 \%$ of Crohn's patients, then it becomes economically more viable."

Among recently approved drugs that target multiple auto-immune diseases is Tecfidera, based on the ester dimethyl fumarate and approved for the treatment of MS in the EU in 2013. It has been heralded as a breakthrough because it can be taken orally, and was earlier approved in Germany for the treatment of psoriasis, an apparently unrelated disease that affects the skin rather than the central nervous system [3]. It is also an example of a drug approved on the basis of its efficacy in clinical trials, rather than because its makers understand precisely how it works. "Its exact mechanism of action is still unknown, but oxidative stress may be involved," commented Bonnie Dittel, Senior Investigator at the Wisconsin Blood Research Institute in the USA and head of a research team focusing on regulation of the auto-immune response.

There are also examples where the development of auto-immune drugs has been halted or delayed for economic reasons, despite promising results in early clinical trials. This was the case for rontalizumab, a monoclonal antibody developed by Roche that targets interferon type 1, one of a group of signaling cytokines that alert neighboring cells to virus infection and instigate various immune responses. Rontalizumab successfully completed phase II trials for treatment of SLE, which attacks multiple organs, and achieved clinical proof of concept. Yet Roche announced in October 2013 that they will not proceed with phase III trials because the compound does not meet economic criteria.

This decision came despite a growing understanding that interferon could be a promising target for the therapeutic treatment of a range of auto-immune diseases, not just Lupus. "I think in Lupus the next thing that will be quite big will be inhibition in the interferon system," Vyse said. "I think that's going to work in a subset of patients and inhibition of interferon will be relevant for a number of other diseases as well. That pathway is dis-regulated in a number of different auto-immune diseases."

$\mathrm{T}$ he finding that the same drug can work against multiple diseases suggests that the whole approach to developing and approving therapies for specific targets should be revised, Cotsapas explained. "Right now, for very good reasons, we don't use therapies developed for one disease on another disease, because doctors prescribe based on symptoms and if a medicine is approved for use in rheumatoid arthritis, there is no reason that a neurologist treating an MS patient will prescribe it. It doesn't make sense. But it may make perfect sense if it's hitting the same pathway partially underlying both diseases and a given patient has a deficit in that pathway," he said.

\section{"The finding that the same drug can work against multiple diseases suggests that the whole approach to developing and approving therapies for specific targets should be revised"}

However, discovering single drugs that effectively treat a wide range of autoimmune diseases will not mean that all such diseases become treatable. A clear example is type 1 diabetes, the symptoms of which occur only after the immune system has already destroyed most insulin-producing cells. "Since type 1 diabetes is largely diagnosed after beta cell destruction has reached a critical point, it cannot be treated with immune modifiers at that stage," Dittel 
explained. Until cell-based therapies become available to regrow these beta cells, there is no point in modifying immune responses to treat diabetes, and insulin remains the only viable option. Even for auto-immune diseases that do share common underlying pathways, a single drug to treat both may not be possible, as they can require quite different therapeutic approaches. "In MS, if you can prevent or diminish the $\mathrm{T}$ cell response, the disease will be tempered," Dittel said. "Then RA has a large inflammatory component that is driven in part by TNF- $\alpha$, a major pro-inflammatory molecule that can be targeted quite successfully."

Overall, a better understanding of the underlying mechanisms, pathways, and molecules involved in auto-immune diseases should lead to the better use of existing drugs and the development of new therapies to modify the action of specific target pathways. On the research side, such knowledge will help to unify the understanding of autoimmune diseases, while on the clinical side, it will give physicians more options to treat patients based on their individual molecular deficiencies. The ultimate goal, however, remains the discovery of real cures for autoimmune diseases, not just therapies for managing their progress or symptoms.

\section{References}

1. Feldmann M, Maini RN (2003) Lasker Clinical Medical Research Award. TNF defined as a therapeutic target for rheumatoid arthritis and other autoimmune diseases. Nat Med 9: $1245-1250$

2. Cotsapas C, Hafler DA (2013) Immunemediated disease genetics: the shared basis of pathogenesis. Trends Immunol 34: $22-26$

3. Mrowietz U, Altmeyer P, Bieber T, Röcken $M$, Schopf RE, Sterry W (2007) Treatment of psoriasis with fumaric acid esters (Fumaderm ${ }^{\circledR}$ ). JDDG 5: $716-717$

4. Feller M, Huwiler K, Schoepfer A, Shang A, Furrer H, Egger M (2010) Long-term antibiotic treatment for Crohn's disease: systematic review and meta-analysis of placebo-controlled trials. Clin Infect Dis 50: $473-480$ 